\title{
Anti-tumor activity of Sann-Joong-Kuey-Jian-Tang alone and in combination with 5-fluorouracil in a human colon cancer colo 205 cell xenograft model
}

\author{
CHUN-YUAN CHENG ${ }^{1,2}$, YI-HSIANG LIN ${ }^{3}$ and CHIN-CHENG SU ${ }^{3,4}$ \\ ${ }^{1}$ Institute of Medicine, Chung Shan Medical University, Taichung 40201; ${ }^{2}$ Changhua Christian Hospital, Changhua 500; \\ ${ }^{3}$ Institute of Pharmacology and Toxicology, Tzu-Chi University; ${ }^{4}$ Division of General Surgery, \\ Buddhist Tzu-Chi General Hospital, Hualien 97004, Taiwan, R.O.C.
}

Received September 29, 2009; Accepted November 27, 2009

DOI: $10.3892 / \mathrm{mmr}+00000244$

\begin{abstract}
Malignant tumors are the leading cause of death in Taiwan; among these, colon cancer ranks third as a cause of cancer-related death. Sann-Joong-Kuey-Jian-Tang (SJKJT), a traditional Chinese medicinal prescription, has been used to treat lymph node diseases and infectious lesions, and exhibits cytotoxic activity in many cancer cell lines. Our previous studies demonstrated that SJKJT inhibits the proliferation of human colon cancer colo 205 cells in vitro. The aim of this study was to evaluate the anti-tumor activity of SJKJT alone and in combination with 5-fluorouracil (5-FU) in vivo. SCID mice bearing human colon cancer colo 205 cell xenografts were administered SJKJT alone (30 mg/kg daily, p.o.), SJKJT (30 mg/kg daily, p.o.) in combination with $5-\mathrm{FU}(30 \mathrm{mg} / \mathrm{kg}$ weekly, i.p.), or vehicle alone. At the end of the 4-week dosing schedule, the tumor and animal body weights were individually measured. The SCID mice were sacrificed with $\mathrm{CO}_{2}$ inhalation, the xenograft tumors were dissected, and the protein expression of microtubule-associated protein light chain 3 (MAP-LC3-II) in colo 205 xenograft tumors was measured by Western blotting. In the control, SJKJT-, and SJKJT plus 5-FU-treated mice, the tumor weights were $6.37 \pm 2.57,0.43 \pm 0.35$ and $1.63 \pm 0.46 \mathrm{~g}$, and the mice body weights were $29 \pm 0.55,29 \pm 2.71$ and $27 \pm 0.77 \mathrm{~g}$, respectively. Treatment with SJKJT resulted in a reduction in tumor weight compared with the control group, indicating that SJKJT inhibits tumor growth in a colo 205 xenograft model. SJKJT also increased LC3-II protein expression as compared to the controls. The present study shows that SJKJT alone or in combination with 5-FU has a positive effect on the treatment of SCID mice bearing human colon cancer colo 205 cell xenografts. This suggests that SJKJT has therapeutic potential in the treatment of human colon cancer.
\end{abstract}

Correspondence to: Dr Chin-Cheng Su, Division of General Surgery, Buddhist Tzu Chi General Hospital, No 707, Sec. 3, Chung Yang Road, Hualien 970, Taiwan, R.O.C.

E-mail: succ.maeva@msa.hinet.net

Key words: Sann-Joong-Kuey-Jian-Tang, 5-fluorouracil, colo 205 cell xenograft model, microtubule-associated protein light chain 3

\section{Introduction}

Traditional Chinese medicine, which is founded on over 5,000 years of tradition and employs an enormous variety of drugs of plant origin, is a well-known medical practice in Asia (1). Sann-Joong-Kuey-Jian-Tang (SJKJT) is a traditional Chinese medicine that has been prescribed to treat diseases with lymphadenopathy and inflammation or tumors with lymph node invasion. Colon cancer is the second leading cause of cancer-related death in Western societies (2). In Taiwan, it is ranked third as the principal cause of cancer-related death (3), and has been treated with SJKJT as a complementary medicine. Our previous studies showed that SJKJT inhibits the proliferation of colo 205 cells and up-regulates the protein expression of microtubule-associated protein light chain 3 (MAP-LC3-II) in vitro (4). However, the protein expression of MAP-LC3-II in human colon cancer treated with SJKJT in vivo is in need of further investigation. 5-Fluorouracil (5-FU) is one of the most commonly used chemotherapeutic agents in the treatment of colon cancer, but has limited efficacy (5). In the present study, we evaluated the anti-tumor efficacy of SJKJT in combination with 5-FU in a human colon cancer colo 205 cell xenograft model, and assayed the protein expression of MAP-LC3-II in these tumors.

\section{Materials and methods}

Chemicals and reagents. Fetal bovine serum (FBS), sodium pyruvate, HEPES, dimethyl sulfoxide (DMSO), RPMI-1640 and the antibodies to MAP-LC3-II and $\beta$-actin were obtained from Sigma-Aldrich (St. Louis, MO, USA). Penicillinstreptomycin, trypsin-EDTA and glutamine were obtained from Gibco BRL (Grand Island, NY, USA). Sodium dodecyl sulfate polyacrylamide gel electrophoresis (SDS-PAGE) running buffer (10X), Tris, Tween-20, SDS and 5X TBE buffer were obtained from Amresco (St. Louis, MO, USA). BioMax Film was obtained from Kodak.

SJKJT consists of 17 species of medicinal herbs: Coptis chinensis Franch, Cimicifuga heracleifolia Komar, Scutellaria baicalensis Georgi, Gentiana scabra Bunge, Trichosanthes cucumeroides Maxim, Phellodendron amurense Rupr, Anemarrhena asphodeloides Bunge, Platycodon grandiflorum, 


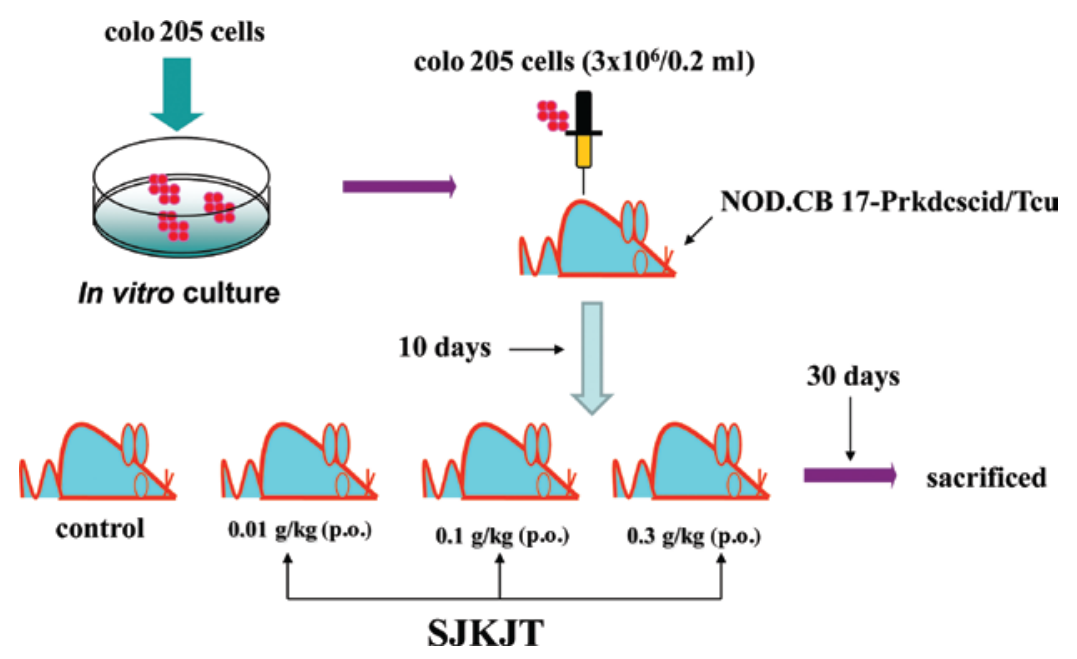

Figure 1. First experimental design. Three-week-old male nude SCID mice were xenografted with colon cancer colo 205 cells $\left(3 \times 10^{6} / 0.2 \mathrm{ml}\right)$, divided randomly into four groups and maintained in a pathogen-free environment. From day 10, SJKJT (dissolved in normal saline) was administered orally at concentrations of $0.01,0.1$ and $0.3 \mathrm{mg} / \mathrm{kg}$ daily for 30 days. As a control, mice were treated with normal saline $\left(0.1 \mathrm{ml} / 10 \mathrm{~g}\right.$ body weight). Mice were sacrificed by $\mathrm{CO}_{2}$ inhalation.

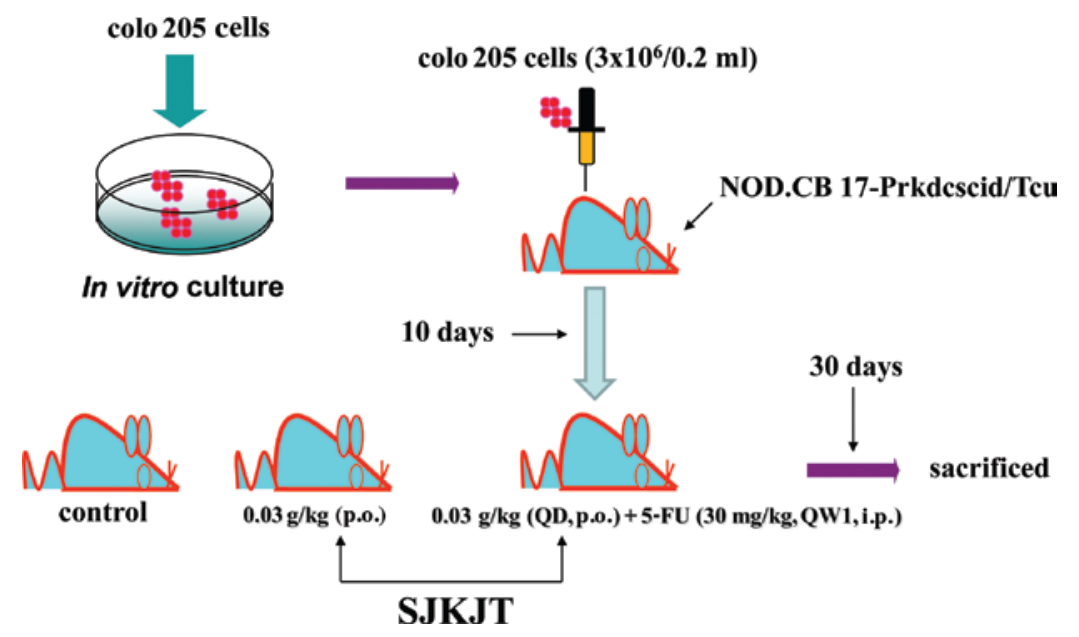

Figure 2. Second experimental design. Three-week-old male nude SCID mice were xenografted with colon cancer colo 205 cells $\left(3 \times 10^{6} / 0.2 \mathrm{ml}\right)$, divided randomly into three groups and maintained in a pathogen-free environment. From day 10, the mice were administered SJKJT [30 mg/kg daily (QD), p.o.], a combination of SJKJT (30 mg/kg daily, p.o.) and 5-FU [30 mg/kg weekly (QW1), i.p.], or vehicle (normal saline) alone. At the end of the 4-week dosing schedule, mice were sacrificed by $\mathrm{CO}_{2}$ inhalation.

Laminaria japonica Aresch, Bupleurum scorzoneri folium Willd (Bupleurum chinese DC), Glycyrrhiza uralensis Fisch, Sparganium toloniferum Buch, Curcuma aeruginosa Roxb, Forsythia suspense Vahl, Pueraria lobata Ohwi, Paeonia lactiflora Pall and Angelica sinensis Diels (6). Crude extract of SJKJT was obtained from Chuang Song Zong Pharmaceutical Co., Ltd. (Ligang Plant, Taiwan).

Cell culture. Human colon adenocarcinoma colo 205 cells were obtained from the Food Industry Research and Development Institute (Hsin-chu, Taiwan). Cells were grown in $75-\mathrm{cm}^{3}$ tissue culture flasks at $37^{\circ} \mathrm{C}$ in a humidified atmosphere of $5 \%$ $\mathrm{CO}_{2}$ and $95 \%$ air in RPMI-1640 medium containing $10 \%$ heatinactivated FBS, $2 \%$ penicillin-streptomycin $(10,000 \mathrm{U} / \mathrm{ml}$ penicillin; $10 \mathrm{mg} / \mathrm{ml}$ streptomycin), $1 \%$ HEPES, $1 \%$ sodium pyruvate and $1 \%$ glutamine.

Experimental design. In our first experiment, 3-week-old male nude SCID mice were xenografted with colon cancer colo 205 cells $\left(3 \times 10^{6} / 0.2 \mathrm{ml}\right)$, divided randomly into four groups and maintained in a pathogen-free environment at the Laboratory Animal Center of Tzu Chi University, Hualien, Taiwan. As of day 10, SJKJT (dissolved in normal saline) was administered orally at concentrations of $0.01,0.1$ and $0.3 \mathrm{~g} / \mathrm{kg}$ daily for 30 days. As a control, mice bearing xenograft tumors were treated separately with normal saline $(0.1 \mathrm{ml} / 10 \mathrm{~g}$ body weight). Mice were sacrificed by $\mathrm{CO}_{2}$ inhalation, then the xenograft tumors were dissected and individually weighed, and proteins were extracted for Western blot analysis (Fig. 1).

In the second experiment, 3-week-old male nude SCID mice were xenografted with colon cancer colo 205 cells $(3 \times 10 \%$ $0.2 \mathrm{ml}$ ), divided randomly into three groups and maintained in a pathogen-free environment (Laboratory Animal Center of Tzu Chi University). From day 10, the mice were administered SJKJT (30 mg/kg daily, p.o.), a combination of SJKJT (30 mg/ $\mathrm{kg}$ daily, p.o.) and 5-FU (30 mg/kg weekly, i.p.), or vehicle alone (normal saline). At the end of the 4-week dosing schedule, the SCID mice were sacrificed by $\mathrm{CO}_{2}$ inhalation, then the 


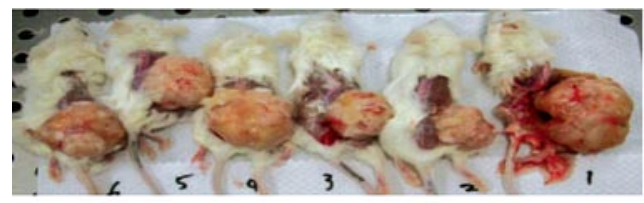

Control

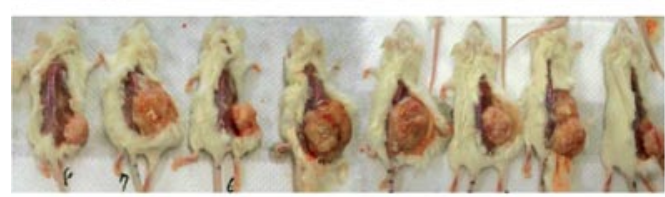

$0.01 \mathrm{~g} / \mathrm{kg}$

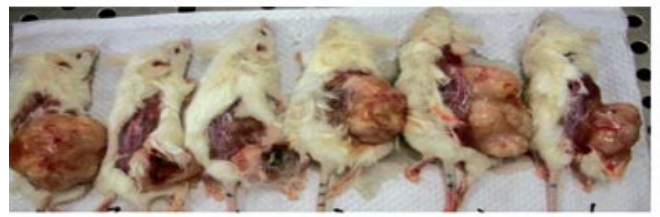

$0.1 \mathrm{~g} / \mathrm{kg}$

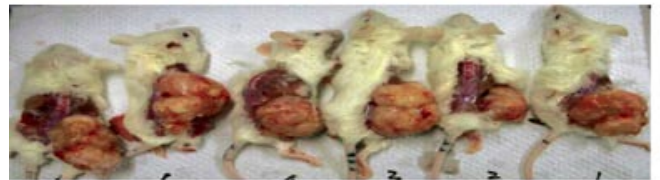

$0.3 \mathrm{~g} / \mathrm{kg}$

Figure 3. Dissected colo 205 cell xenograft tumors. Mice were treated for 30 days with normal saline only (control) or with SJKJT concentrations of $0.01,0.1$ and $0.3 \mathrm{~g} / \mathrm{kg}$ daily, then sacrificed by $\mathrm{CO}_{2}$ inhalation. The colo 205 cell xenograft tumors were then dissected.

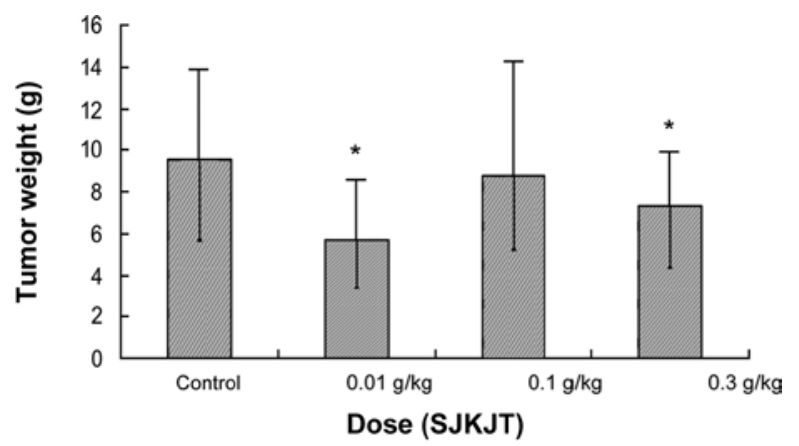

Figure 4. Colo 205 cell xenograft tumor weights. Mice were treated for 30 days with normal saline only (control) or with SJKJT concentrations of $0.01,0.1$ and $0.3 \mathrm{~g} / \mathrm{kg}$ daily, and the colo 205 cell xenograft tumors were dissected and individually weighed.

xenograft tumors were dissected and individually weighed, and proteins were extracted for Western blot analysis (Fig. 2).

Determination of protein expression by Western blot analysis. MAP-LC3-II as well as $\beta$-actin proteins were extracted as previously described (7). Briefly, the xenograft tumors were dissected, and the thick liquid was resuspended in modified Pro-prep ${ }^{\text {TM }}$ buffer (Intron Biotechnology) for $40 \mathrm{~min}$ at $4^{\circ} \mathrm{C}$. Lysates were immediately centrifuged at $13,000 \mathrm{x}$ g for $20 \mathrm{~min}$ at $4^{\circ} \mathrm{C}$, and the supernatant was collected, aliquoted ( $20 \mu \mathrm{l} /$ tube) and stored at $-80^{\circ} \mathrm{C}$ until assay. The extracted protein concentrations were determined using the Bradford method (8). Protein samples were separated by $8 \%$ ( $\beta$-actin) or $15 \%$ (MAP-LC3-II) sodium dodecyl sulfate polyacrylamide gel electrophoresis (SDS-PAGE) as previously described (9). The SDS-separated proteins were equilibrated in transfer buffer $(25 \mathrm{mM}$ Tris, $\mathrm{pH}$ 8.5, 0.2 M glycine and 20\% methanol) and transferred to

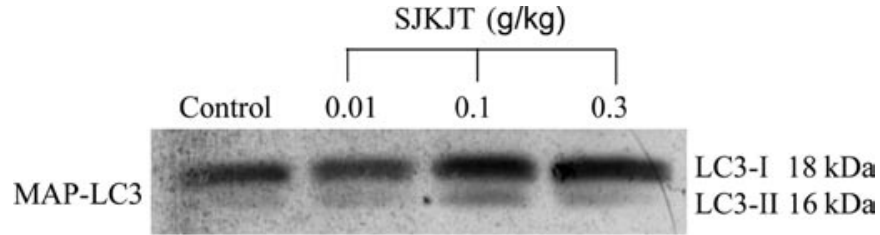

$\beta$-actin
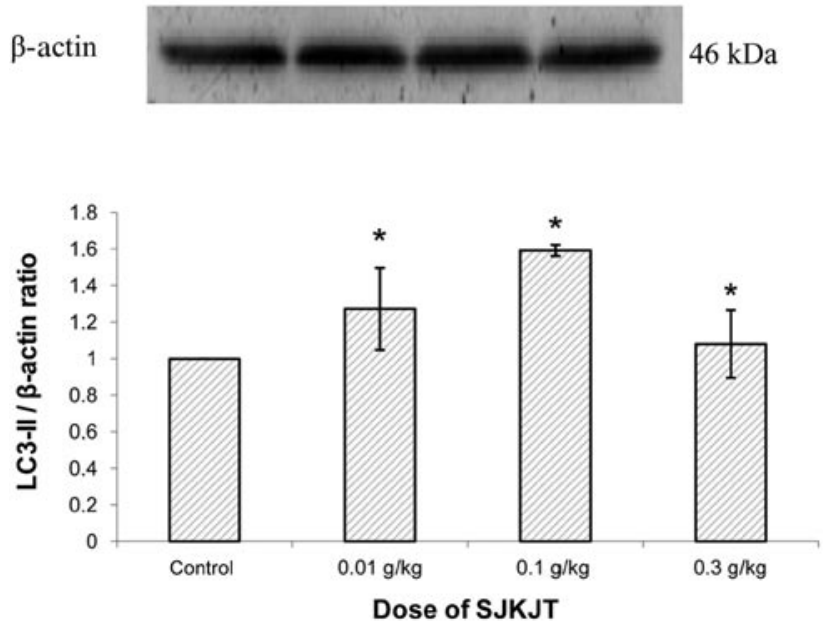

Figure 5. Protein expression of MAP-LC3-II in SCID mice with colo 205 cell xenograft tumors treated with SJKJT concentrations of $0.01,0.1,0.3 \mathrm{~g} / \mathrm{kg}$ daily. The tumors were dissected, and the proteins were extracted for Western blot analysis. SJKJT up-regulated the protein expression of MAP-LC3-II as compared to the controls. Values are expressed as the mean $\pm \mathrm{SD}$. ${ }^{*} \mathrm{P}<0.05$, significant difference compared to the controls.

PVDF membranes (Millipore Corp., Bedford, MA, USA). The membranes were incubated with $5 \%$ nonfat dry milk in Trisbuffered saline containing $0.1 \%$ Tween-20 for $1 \mathrm{~h}$, then washed and incubated with appropriate dilutions of specific antibodies, including anti-AP-LC3-II (1:1000) and anti- $\beta$-actin $(1: 15000)$ (Sigma-Aldrich), at $4^{\circ} \mathrm{C}$ overnight. Following incubation with anti-mouse peroxidase-conjugated antibody (1:15000) (SigmaAldrich), the immunoreactive bands were visualized with an enhanced chemiluminescence (ECL) detection kit (Millipore Corp.). $\beta$-actin levels were used as an internal control for Western blotting. Immunoreactive bands were scanned (GS-800; Bio-Rad Life Science Products, Hercules, CA, USA) and analyzed using a digital scanning densitometer (Quantity One, v4.4.0, Bio-Rad Life Science Products).

Statistical analysis. Values are presented as the mean \pm SD. The Student's t-test was used to analyze statistical significance. A p-value $<0.05$ was considered statistically significant.

\section{Results}

Effect of SJKJT on colo 205 cell xenograft tumors. In the first experiment, the growth of colo 205 cell xenograft tumors was found to be inhibited in SCID mice treated daily for 30 days with normal saline or SJKJT concentrations of 0.01, 0.1, $0.3 \mathrm{~g} /$ $\mathrm{kg}$ (Fig. 3). SJKJT at a low dose $(0.01 \mathrm{~g} / \mathrm{kg}$ daily) inhibited the growth of colo 205 cell xenograft tumors significantly compared with the higher doses (0.1 and $0.3 \mathrm{~g} / \mathrm{kg}$ daily) (Fig. 4).

According to the results of Western blotting, the protein expression of MAC-LC3-II in the SJKJT-treated xenograft tumors was up-regulated as compared to the controls (Fig. 5). 


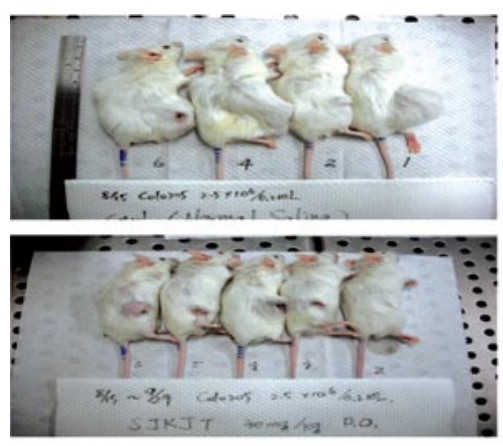

A

Control group

N/S p.o./QD; N/S i.p./QW1

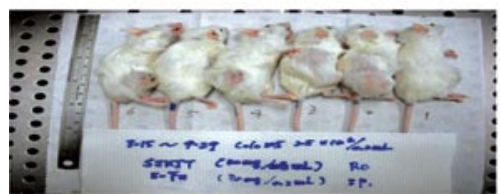

SJKJT (30 mg/kg) p.o./QD N/S i.p./QW1

SJKJT $(30 \mathrm{mg} / \mathrm{kg})$ p.o./QD $+5-\mathrm{FU}(30 \mathrm{mg} / \mathrm{kg})$ i.. . $/ \mathrm{QW} 1$

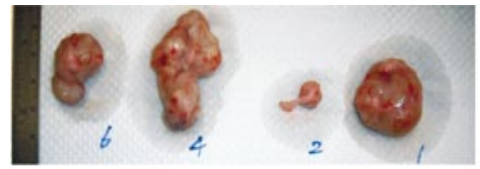

B

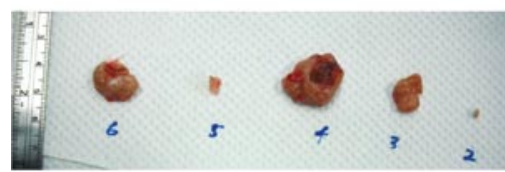

SJKJT ( $30 \mathrm{mg} / \mathrm{kg})$ p.o./QD N/S i.p./QW1

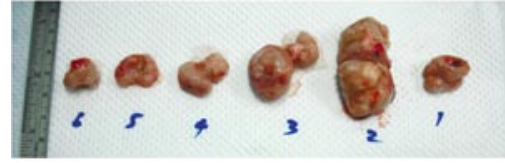

SJKJT (30 mg/kg) p.o./QD $+5-F U(30 \mathrm{mg} / \mathrm{kg})$ i.p. $/ \mathrm{QW} 1$

Figure 6. Dissected colo 205 cell xenograft tumors. Mice were treated for 30 days with normal saline (N/S) only (control), SJKJT [30 mg/kg daily (QD), p.o.], or SJKJT (30 mg/kg daily, p.o.) plus 5 -FU [30 mg/kg weekly (QW1), i.p.], sacrificed by $\mathrm{CO}_{2}$ inhalation (A) and the colo 205 cell xenograft tumors were dissected (B).

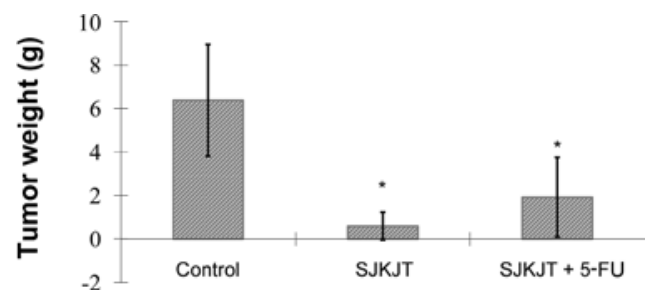

Figure 7. Colo 205 cell xenograft tumor weights. SCID mice with colo 205 cell xenograft tumors treated with normal saline only (control), SJKJT (30 mg/kg daily, p.o.), or SJKJT (30 mg/kg daily, p.o.) plus 5-FU (30 mg/kg weekly, i.p.). The tumors were dissected and weighed.

Effect of SJKJT versus SJKJT plus 5-FU on colo 205 cell xenograft tumors. In the second experiment, the growth of colo 205 cell xenograft tumors treated with SJKJT $(30 \mathrm{mg} /$ $\mathrm{kg}$ daily, p.o.) or a combination of SJKJT (30 mg/kg daily, p.o.) and 5-FU (30 mg/kg weekly, i.p.) was inhibited by 93.25 and $74.41 \%$, respectively, as compared to the vehicle (normal saline) only controls. In the control, SJKJT-, and SJKJT plus 5-FU-treated SCID mice, the weights of the colo 205 cell xenograft tumors were $6.37 \pm 2.57,0.43 \pm 0.35$ and $1.63 \pm 0.46 \mathrm{~g}$ (Fig. 6), and the mice body weights were $29 \pm 0.55,29 \pm 2.71$
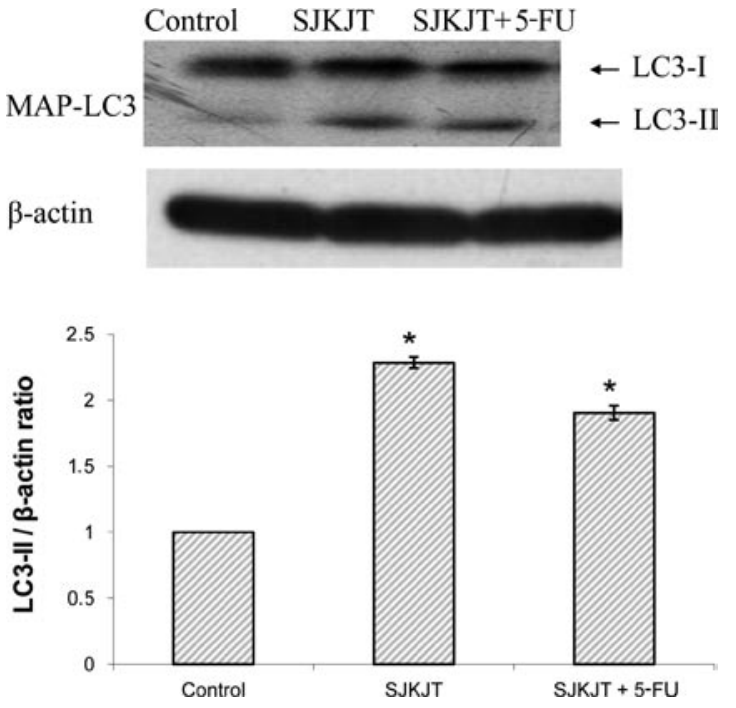

Figure 8. Protein expression of MAP-LC3 II in SCID mice with colo 205 cell xenograft tumors treated with normal saline only (control), SJKJT (30 mg/ $\mathrm{kg}$ daily, p.o.) or SJKJT (30 mg/kg daily, p.o.) plus 5-FU (30 mg/kg weekly, i.p.). Tumors were dissected, and the proteins extracted for Western blot analysis. SJKJT and SJKJT plus 5-FU up-regulated the protein expression of MAP-LC3 II as compared to the controls. Values are expressed as the mean \pm $\mathrm{SD} .{ }^{*} \mathrm{P}<0.05$, significant difference compared to the controls.

and $27 \pm 0.77 \mathrm{~g}$, respectively (Fig. 7). These results indicate that SJKJT alone or in combination with 5-FU has a positive effect on the human colon cancer colo 205 cell xenografts, although SJKJT alone is more effective than SJKJT in combination with 5-FU.

Additionally, the results of Western blotting showed that SJKJT, as well as SJKJT plus 5-FU, up-regulated the protein expression of MAP-LC3-II compared to the controls (Fig. 8).

\section{Discussion}

It has been demonstrated that the examination of plant extracts used in traditional Chinese medicine may lead to the identification of novel active constituents (10), and the combination of traditional Chinese medicine and modern molecular medicine approaches has the potential to contribute to the development of the future practice of cancer medicine (11).

It is well documented that autophagy is one means of programmed cell death, and that targeting the autophagic pathway may be utilized to improve cancer therapy $(12,13)$. MAP-LC3-II protein expression has been used as a marker of autophagy, and can be detected using Western blot analysis $(14,15)$.

In a previous study, we demonstrated that SJKJT inhibited proliferation in human colon cancer colo 205 cells in a dosedependent manner in vitro. (4). The results of the present study confirm that SJKJT (30 mg/kg daily, p.o.), as well as SJKJT (30 mg/kg daily, p.o.) plus 5-FU (30 mg/kg weekly, i.p.), inhibit colo 205 cell xenograft tumor growth in vivo, although SJKJT alone was more effective than SJKJT plus 5-FU. In both the experiments conducted, an increase in the protein expression of MAP-LC3-II as compared to the control was observed. Therefore, one of the molecular mechanisms behind 
the inhibition of proliferation in colo 205 cell xenografts may be the up-regulation of MAP-LC3-II protein expression.

In a previous study, we showed that SJKJT induced apoptosis in colon cancer cells by up-regulating Fas, TNF- $\alpha$, Caspase- 8 and Caspase- 3 protein expression in vitro and in vivo (16). Together, these findings suggest that SJKJT has therapeutic potential in the treatment of human colon cancer.

\section{Acknowledgements}

This study was supported by grant no. TCRD97-05 from the Research Section of the Buddhist Tzu Chi General Hospital, Hualien, Taiwan, R.O.C.

\section{References}

1. Rauh R, Kahl S, Boechzelt H, Bauer R, Kaina B and Efferth T: Molecular biology of cantharidin in cancer cells. Chin Med 2: 8, 2007.

2. Jemal A, Tiwari RC, Murray T, et al: Cancer statistics, 2004. CA Cancer J Clin 54: 8-29, 2004.

3. Statistics of Causes of Death, 2007. Department of Health, Executive Yuan, Taipei, Taiwan R.O.C, p33, 2008.

4. Cheng CY, Lin YH and Su CC: Sann-Joong-Kuey-Jian-Tang increases the protein expression of microtubule-associated protein II light chain 3 in human colon cancer colo 205 cells. Mol Med Rep 2: 707-711, 2009.

5. James K, Eisenhauer E, Christian M, Terenziani M, Vena D and Mudal A: Measuring response in solid tumors: unidimensional versus bidimensinal measurement. J Natl Cancer Inst 91: 523-528, 1999 .
6. Hsu YL, Yen MH, Kuo PL, et al: San-Zhong-Kui-Jian-Tang, a traditional Chinese medicine prescription, inhibits the proliferation of human breast cancer cells by blocking cell cycle progression and inducing apoptosis. Biol Pharm Bull 29: 2388-2394, 2006.

7. Su CC and Lin YH: Tanshinone IIA inhibits human breast cancer cells through increased Bax to Bcl-xL ratios. Int J Mol Med 22: 357-361, 2008.

8. Bradford MM: A rapid and sensitive method for the quantitation of microgram quantities of protein utilizing the principle of protein-dye binding. Anal Biochem 72: 248-254, 1976.

9. Chen HC, Hsieh WT, Chang WC and Chung JG: Aloe-emodin induced in vitro $\mathrm{G} 2 / \mathrm{M}$ arrest of cell cycle in human promyelocytic leukemia HL-60 cells. Food Chem Toxicol 42: 1251-1257, 2004.

10. Efferth T, Kahl S, Paulus K, et al: Phytochemistry and pharmacogenomics of natural products derived from traditional Chinese medicine and Chinese materia medica with activity against tumor cells. Mol Cancer Ther 7: 152-161, 2008.

11. Parekh HS, Liu G and Wei MQ: A new dawn for the use of traditional Chinese medicine in cancer therapy. Mol Cancer 8: $1-8,2009$

12. Clarke PG: Developmental cell death: morphological diversity and multiple mechanisms. Anat Embryol 181: 195-213, 1990

13. Moretti Lu, Yang ES, Kim KW and Lu B: Autophagy signaling in cancer and its potential as novel target to improve anticancer therapy. Drug Resist Updat 10: 135-143, 2007.

14. Kirkegaard K, Taylor MP and Jackson WT: Cellular autophagy: surrender, avoidance and subversion by microorganisms. Nat Rev Microbiol 2: 301-314, 2004.

15. Eskelinen EL, Prescott AR, Cooper J, et al: Inhibition of autophagy in mitotic animal cells. Traffic 3: 878-893, 2002.

16. Cheng CY, Lin YH and Su CC: Sann-Joong-Kuey-Jian-Tang up-regulates the protein expression of Fas and TNF- $\alpha$ in colo 205 cells in vivo and in vitro. Mol Med Rep 3: 63-67, 2010. 